\title{
Study on Sound Field Distribution Rule for Tank Structures of Large Oil-immersed Transformers
}

\author{
Ma Yuchao ${ }^{1 *}$, Mo Juan ${ }^{1}$, Yu Jinshan ${ }^{2}$, Li Xiang ${ }^{1}$ and Zheng Zhongyuan ${ }^{2}$ \\ ${ }^{1}$ China Electric Power Research Institute, 100055, Beijing, China \\ ${ }^{2}$ State Grid Tianjin Electric Power Research Institute, 300191, Tianjin, China
}

\begin{abstract}
Large oil-immersed transformers are an important part of the transmission and distribution network in power systems. Power transformers are the main noise source of substations. Because of the uneven manufacturing process, aging equipment, long-term operation, and close distance from sensitive points, the problem of transformer noise pollution has become increasingly prominent. In this paper, the transmission and analysis model is established for transformer sound waves on the interface between insulating oil and tank body according to the sound wave propagation rule in complicated medium, and the simplified acoustic simulation model is constructed for large oil-immersed transformers by simulating the vibration noise of transformer core with monopole sound source, with which, the sound field distribution rule inside and outside the transformer tank structure is obtained, and finally, the influence factors for noise distribution are given. The results of the study provide control basis for reducing transformer noise.
\end{abstract}

\section{Introduction}

Transformers are the major noise source for substations, and its vibration and noise generation mechanism as well as the corresponding reduction and noise reduction technology have been the study focus in relevant areas. In order to grasp the characteristics and propagation rules for vibration and noise of the transformers, relevant research institutes at home and abroad have made indepth study on the vibration mechanism and noise characteristics of transformer core and winding by theoretical analysis, tests and experiments as well as numerical simulation technology and have obtained a series of study results in terms of the electromagnetic vibration mechanism, vibration and noise test technology of the transformers. The vibration noise from the core and winding of large oil-immersed transformers are mainly transferred to the tank structure via internal structural parts and insulating oil; it produces noise radiation outward. In insulating oil, part of the vibration from core and winding are directly transferred to the insulating oil to form a stable reverberation field in the oil and act on the tank structure as noise load. However, the study on sound field distribution inside and outside the insulating oil of large oil-immersed transformers is insufficient, no simulation analysis and measured data of the transformers are provided, and the qualitative and quantitative analysis on the influencing factor of noise is inadequate. In this paper, the transmission and analysis model is established for transformer sound wave on the interface between insulating oil and tank body according to the sound wave propagation rule in complicated medium on the basis of the study above, and the simplified acoustic simulation model is constructed for large oil-immersed transformers by simulating the vibration noise of transformer core with monopole sound source, with which, the mechanism for the internal and external vibration noise from transformer oil acting on the tank structure is revealed, and the sound field distribution rule inside and outside the transformer tank structure is obtained. This paper provides the theoretical basis and data support for vibration and noise reduction methods for tank structure of the transformers.

\section{Noise Generation Mechanisms}

During actual operation, vibration of transformer body is the synthetic effect of three kinds of vibration, including vibration from core, winding and cooling system as well as the process transferring the vibration from core and winding outward via tank structure of the transformers. It is a complex problem. The core vibrates based on a fundamental frequency of $100 \mathrm{~Hz}$, and also due to the mutual frictional and vibration from the silicon steel sheet of the core, the core can generate a vibration with a maximum frequency of $500 \mathrm{~Hz}$, with rare vibration of higher frequency. The fundamental frequency of winding is $100 \mathrm{~Hz}$ as well, and there is almost no other component vibration. The vibration from the acting force from leakage magnetic field on tank shell is also $100 \mathrm{~Hz}$. In consideration of the non-dispersive vibration from several vibration sources, viz. no new frequency component will be generated after vibration superposition, so the integral vibration frequency of transformers is $100 \mathrm{~Hz}$ and its integral multiple and is generally not more than $500 \mathrm{~Hz}$. Figure 1 shows the

\footnotetext{
*Corresponding author: myc2011bjut@ $163 . c o m$
} 
typical distribution of integral vibration spectrum of the transformers (with normalized amplitude). During normal operation of the transformers, the voltage basically remains unchanged, while the load current changes greatly and may contain a harmonic wave. Therefore, the current is the major factor affecting vibration during actual operation of the transformers. In the light of air self-cooling transformers, the large-area radiator suspended outside the transformers is also one of the main factors affecting the vibration. In case the self-resonant frequency of some structures is $100 \mathrm{~Hz}-500$ $\mathrm{Hz}$, resonance may certainly be generated, and then the vibration from the transformers will be big accordingly. The resonance must be absolutely avoided in the design of tank structure for the transformers.

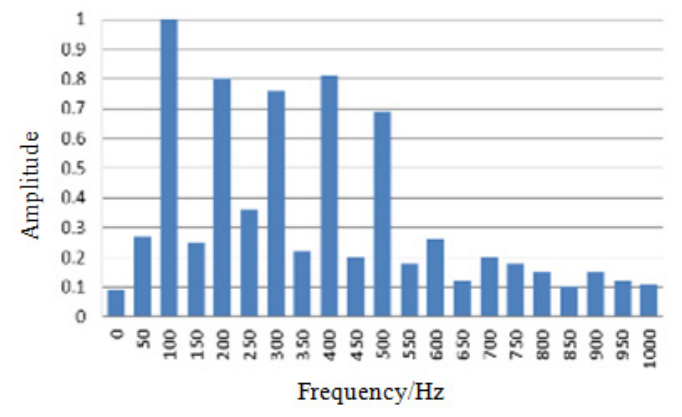

Figure 1. Vibration Spectrum of Typical Single-phase Transformer Tank

\section{Sound Wave Propagation under Multi- layer Media}

There are multi-layer media inside large oil-immersed transformers, while there will be interaction effect between the media and the reflection/projection of sound waves from medium differences when the sound wave is propagated between different media. Therefore, it is required to put the overall impact from medium impedance, medium stiffness difference and medium parameters on sound propagation into overall consideration during analysis of sound field propagation for the system under complex medium conditions. The simplified diagram of media in transformer tanks is shown in Figure 2.

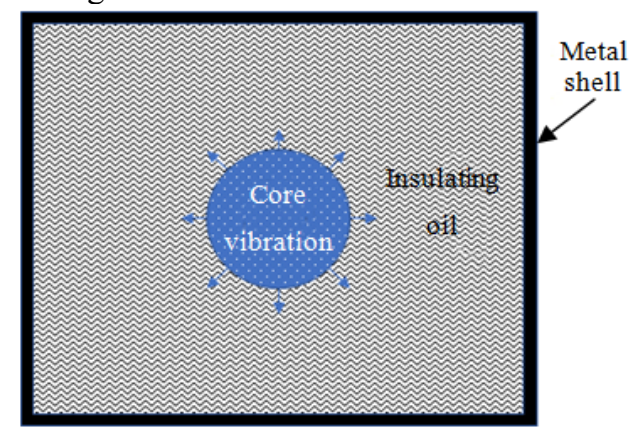

Figure 2. Distribution of Complex Medium in Insulating Oil of the Transformers

Use a two-column format, and set the spacing between the columns at $8 \mathrm{~mm}$. Insert "MATEC Web of Conferences" in even headers and the short form title of the conference in According to the equation for sound propagation between liquid and solid, the propagation of sound field in liquid and its structural dynamics equation should be put into full consideration; the equation for sound propagation in liquid is shown in Formula (1).

$$
\frac{\partial^{2} P}{\partial^{2} t}-c^{2} \nabla^{2} P=\frac{\partial Q}{\partial t}-\frac{\partial F}{\partial x_{i}}+\frac{\partial^{2}\left(T_{i j}\right)}{\partial x_{i} \partial x_{j}}
$$

Where: $P$ refers to sound pressure; it is defined as the sound pressure characteristic value extracted at any position inside the tank, and is represented as sound load at the interface between oil and transformer tank. $c$ indicates the sound velocity, viz. the propagation velocity of sound wave in insulating oil. $\partial Q / \partial t$ is monopole sound source item and is represented as the quality source of sound field changing with time; the vibration of transformer core changes periodically with the time to promote the quality fluctuation of oil, so monopole sound source is used for simulation. $\partial F / \partial x_{i}$ is dipole sound source item, namely, there is gradient acted by external force $F$ in a certain direction of motion by insulating oil; in consideration that the core of the transformers is represented as vibration data at present, the sound source here is $0 . \partial^{2}\left(T_{i j}\right) / \partial x_{i} \partial x_{j}$ is quadrupole sound source item, viz. the acoustic disturbance caused when assuming that the tensor inside insulating oil changes. Presently, its sound source strength is 0 inside insulating oil since there is no disturbance from tensor change inside the insulating oil.

The tank structure will produce vibrations on its surface under the actual function of sound load in the circumstance receiving the sound load excitation from insulating oil, which will result in overall vibration of the transformer tank and next air disturbance around the tank that is propagated outward in sound wave form and furthermore radiation noise. For the vibration dynamic equation of the structure, the load and vibration analysis are shown in Formula (2).

$$
M \dot{x}+C \dot{x}+K x=P(t)
$$

Where, $P(t)$ is the sound load acting on the surface of tank structure, and its load value is input with the results solved by Formula (1). $M$ refers to mass matrix. The tank structure of the transformers is complex and cannot be simulated with independent plate structure, so the discrete finite element matrix is used to represent the mass matrix of system. $C$ indicates damping matrix and is represented as the damping of the structure and the damping characteristics of oil acting on the structure. $K$ refers to stiffness matrix and is represented as the overall stiffness characteristics of the structure.

According to the acoustic wave equation and its surrounding conditions of multi-medium, the Formula (1) should be solved with finite element numerical valuation method, and surface vibration should be used for excitation at sound source boundary. The Helmoholtz equation based on Formula (1) is shown in Figure (3).

$$
\nabla^{2} p(\vec{r}, t)-\frac{1}{c^{2}} \frac{\partial^{2} p(\vec{r}, t)}{\partial t^{2}}=0
$$


The core vibration of the transformers is caused by multiple vibrating bodies, so the vibration characteristics on the surface are complex. In order to simulate the vibration sound source of transformer core easily, equivalent sound source method is used for simulation, the definition of monopole sound source depends on the monopole formula (3) in the transmission and analysis model; the transmission function between vibration and sound needs to be transferred when it is analyzed directly, and the monopole formula should be represented by body velocity. The monopole formula after conversion is shown in Formula (4).

$$
\text { Pow }=\frac{Q^{2} \rho \omega^{2}}{4 \pi c} C
$$

Wherein, Pow refers to sound power, $Q$ indicates body acceleration, $p$ means the density, $c$ is sound velocity, and $w$ refers to angular acceleration. The monopole sound source radiates outward in plane wave type, as shown in Figure 3. The monopole indicates small sound source volume. Generally, the population of sound source in overall system in engineering is of limited scale and supports approximate assumption.

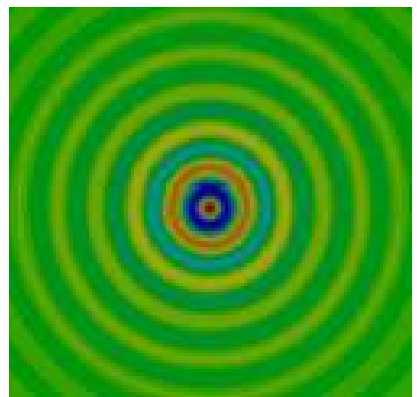

Figure 3. Noise Radiation Type of Monopole

\section{Analysis on the Internal and External Sound Field of Transformer}

Based on the simulation of core vibration using monopole sound source, the influence rule of medium parameters in oil on sound field is obtained by establishing various parameters of insulating oil such as density, sound velocity and temperature. On fluid-solid medium interface, the coupling effect of the bending wave of tank structure is put aside temporarily, and the dynamic characteristics of the structure imposes no direct influence on the propagation and radiation of sound wave.

Simplified monopole sound source is regarded as the excitation boundary to build the simulation model $(3 \mathrm{~m}$ $(\mathrm{L}) \times 1 \mathrm{~m}(\mathrm{~W}) \times 1 \mathrm{~m}(\mathrm{H}))$ of transformer. $6 \mathrm{~mm}$ steel plate is used as the tank structure, and the three kinds of medium, namely insulating oil, steel plate and air are endowed with the impedance boundary of their acoustic propagation characteristics to simulate the propagation characteristics of monopole sound source. The analysis model is shown in Figure 4. The sound field distribution rule of sound waves in three kinds of medium under no propagation of coupled vibration is studied, with the results shown in Figure 5.

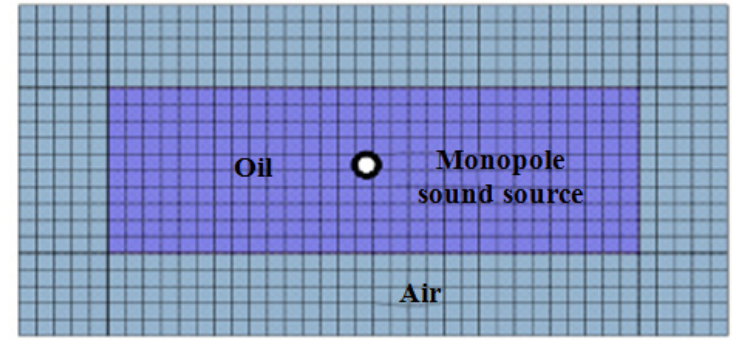

Figure 4. Noise Radiation Type of Monopole

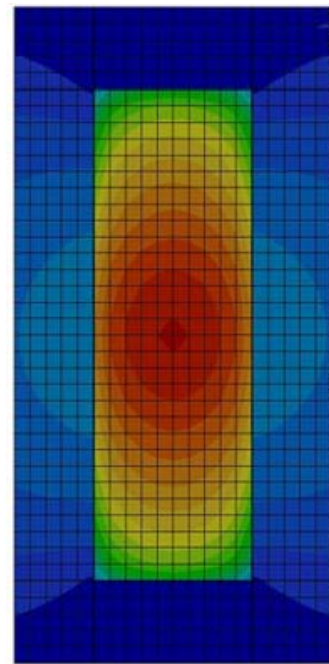

a) $100 \mathrm{~Hz}$ sound field distribution

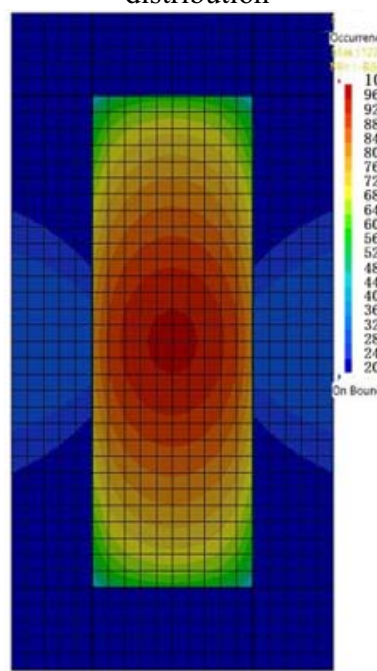

c) $300 \mathrm{~Hz}$ sound field distribution

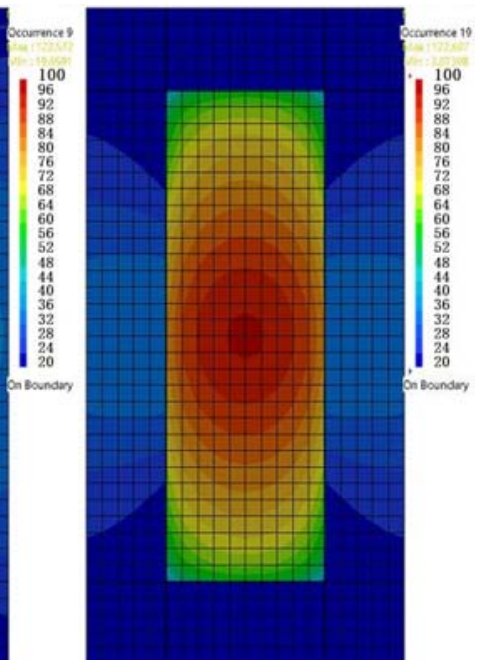

b) $200 \mathrm{~Hz}$ sound field distribution

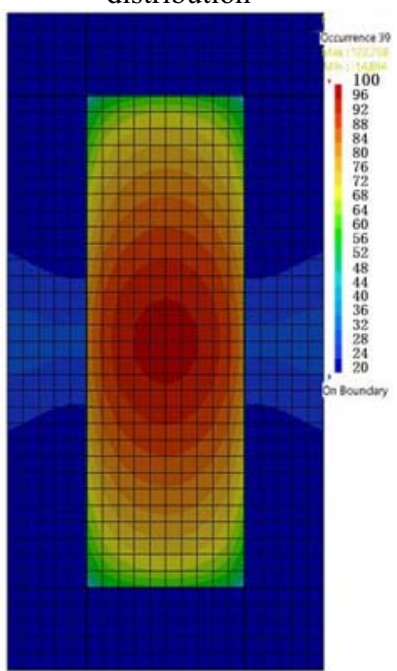

d) $400 \mathrm{~Hz}$ sound field distribution
Figure 5. Propagation Characteristics of Sound Field between Different Media under No Coupling Effect

It can be learnt from Figure 5 that: internal sound field of tank structures of transformers is distributed relatively uniform in varying frequency bands; the distribution space of sound pressure of external sound field reduces and the intensity weakens as the frequency rises, indicating that it is much harder for HF sound wave to penetrate metal wall and enter into the air. Under consistent internal sound source, the internal and external sound pressure curves are shown in Figure 6. Moreover, the following conclusions can be obtained: 
the density of the steel plate is $7,800 \mathrm{~kg} / \mathrm{m} 3$, the thickness is $6 \mathrm{~mm}$, the cross-section mass is $46.8 \mathrm{~kg} / \mathrm{m} 2$ and the sound reduction index between $100 \mathrm{~Hz}-500 \mathrm{~Hz}$ is about $30 \mathrm{~dB}-45 \mathrm{~dB}$; according to the sound pressure difference at both sides, in consideration that internal sound wave is subject to mismatching of acoustic impedance at the interface, the trend embodied by the two is related to the cross-section mass law; and the difference of sound pressure level (SPL) at both sides increases as the frequency rises.

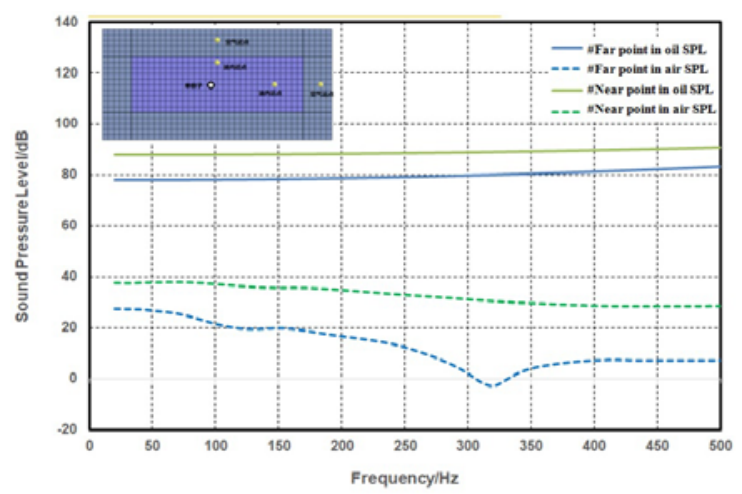

Figure 6. Comparison of SPL Distribution Inside and Outside the Tank

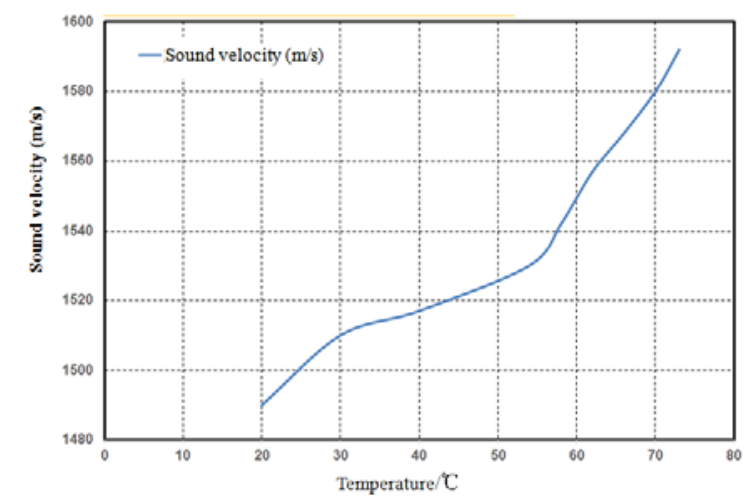

Figure 7. Sound Velocity and Temperature Relation Curve

Based on the testing method of pulse time difference, the sound velocity of insulating oil under different temperatures is obtained, as shown in Figure 7; it can be seen from Figure 7 that the sound velocity difference is controlled within $8 \%$ under different temperature.

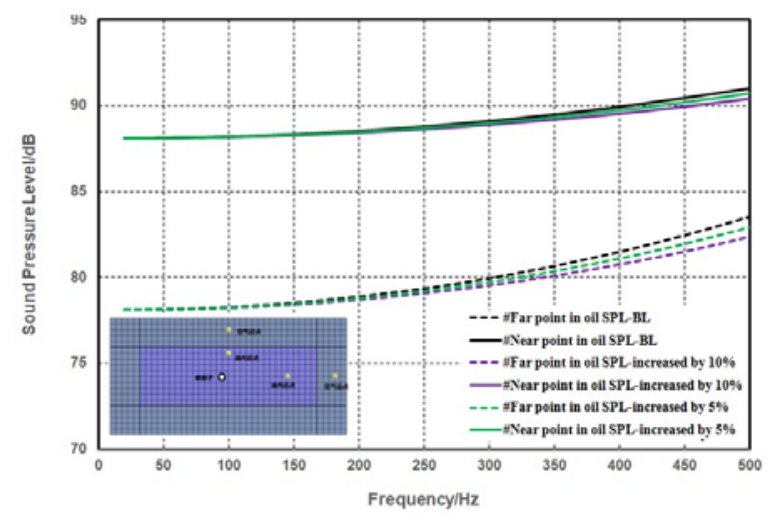

Figure 8. Influence of Sound Velocity in Oil

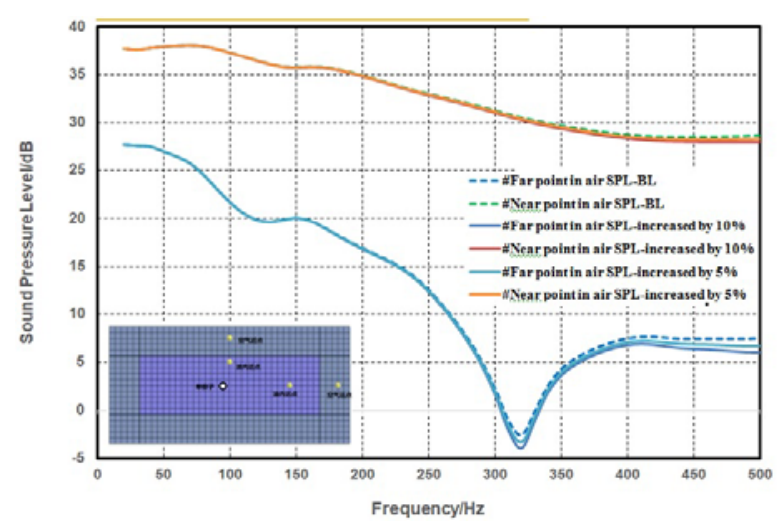

Figure 9. Influence of Sound Velocity Outside the Box

As to sound velocity and temperature of insulating oil, the parameters of insulating oil are modified to study their influences on internal and external sound field; the influences of sound velocity on analysis results are shown in Figure 8 and Figure9; and the following results are obtained based on the analysis results: the sound velocity of transformer oil impose certain influence on internal and external SPL, and the result is that the noise of all frequency points between $300 \mathrm{~Hz}-500 \mathrm{~Hz}$ is within $1.5 \mathrm{~dB}$; its influence is relatively minor under low frequency and expands under high frequency; as the noise of transformer is mainly distributed in low frequency, such influence imposes slight influence on total SPL of transformer, which can be ignored in project.

\section{Conclusion}

With large oil-immersed transformers as the study object, the mechanism of vibration noise in oil-immersed transformers is analyzed in this paper, the sound wave propagation laws in transformer oil under multi-layer media are given, and the propagation analysis model of transformer sound waves at the interface of insulating oil and tank is established. The simplified acoustic simulation model is constructed for large oil-immersed transformers by simulating the vibration noise of transformer core with monopole sound source, the sound field distribution rule inside and outside the transformer tank structure is obtained, and the influence rule of insulating oil parameters of oil-immersed transformer on sound field distribution is mastered. The distribution space of sound pressure of external sound field reduces and the intensity weakens as the frequency rises, indicating that it is much harder for HF sound wave to penetrate metal wall and enter into the air. The insulating oil temperature and sound velocity of transformer imposes certain influence on both internal and external SPL, and its influence is relatively minor under low frequency and expands under high frequency. The conclusions provide theoretical basis and data support for vibration reduction and noise control of transformer tank structures. 


\section{Acknowledgments}

This work was financially supported by Science and Technology project of State Grid Corporation of China. (Study on Key Technology of Noise Reduction for 500 kV Low-noise Transformer Tank Structure Based on Dispersion Theory of Elastic Wave)

Found ID: GCB17201900199.

\section{References}

1. Jing Zheng, Ji Pan, Hai Huang. An experimental study of winding vibration of a single-phase power transformer using a laser doppler vibrometer,Applied Acoustics, 87 (2015).

2. T. Zhao, J. Liang, L. Zou and L. Zhang. A New FXLMS Algorithm With Offline and Online Secondary-Path Modeling Scheme for Active Noise Control of Power Transformers. IEEE Transactions on Industrial Electronics,64 (2017).

3. Mayergoyz ID,Mathematical models of hysteresis and their applications,Academic Press, 2(2003).

4. R. S. Girgis, M. S. Bernesjo,Development of ultralow-noise transformer technologyIEEE Transactions on Power Delivery,26 (2011).

5. Xing Z P, Zhai J Y, Dong S X, et al. Modeling and detection of quasi-static nanotesla magnetic field variations using magnetoelectric laminate sensors, Measurement ence \& Technology,19 (2008).

6. Kim J , Jung Y C . Broadband noise reduction of piezoelectric smart panel featuring negativecapacitive-converter shunt circuit, The Journal of the Acoustical Society of America, 120 (2006).

7. Dahan D,Bilenca A, Eisenstein G, Noise-reduction capabilities of a Raman-mediated wavelength converter,Optics Letters,28 (2003).

8. Fan Zhang, Operational Modal Analysis of Transformer Windings, IEEE Transactions on Power Delivery,35 (2020).

9. Shengchang Ji, Application of Gauss-Newton iteration algorithm on winding radial deformation diagnosis,IEEE Transactions on Power Delivery,34 (2019).

10. Enokizono M,Shimoji H,Ikariga A,et al,Vector magnetic characteristic analysis of electrical machines,IEEE Transactions on Magnetics,41 (2005). 